\title{
Suggestive symptoms and signs of pulmonary thromboembolism in cardiac failure
}

'Faculty of Dental Medicine, University "Ovidius" of Constanta

${ }^{2}$ Faculty of Medicine, University "Ovidius" of Constanta,

\begin{abstract}
Pulmonary thrombembolism can be a complication or it can lead to the onset of heart failure. The early diagnosis of pulmonary thromboembolism may make the difference between life and death. The aim of the study was to determine pulmonary thromboembolism incidence in patients with cardiac failure and to attempt diagnosis optimization.

Study group included 150 patients admitted at Medical Clinic II, Emergency Clinical County Hospital Constanta with preexistent known or unknown heart disease, which led to cardiac failure. Patients were suspected with incipient pulmonary thromboembolism. Dyspnea was the most frequently manifested symptom leading to pulmonary thromboembolism suspicion (99\%). Pulmonary thromboembolism suggestive dyspnea displayed two forms: sudden onset of dyspnea and increased dyspnea in patients suffering from dyspnea at rest. Thoracic pain was present in approximately $90 \%$ of the patients and was manifested in two forms: retrosternal pain, not increasing with respiratory movement, and acute localized thoracic pain, accompanied by hemoptysis and dyspnea; these symptoms suggest pulmonary infarction.
\end{abstract}

\section{Niculescu Zizi}

Faculty of Medicine, University "Ovidius" of Constanta University Street, No. 1, Campus B

Constanta, Romania

e-mail: ziziniculescu@yahoo.co.uk

phone: +40751268420
Pulmonary thromboembolism complicates clinically manifested heart failure or precipitates heart failure onset in patients with latent myocardial dysfunction.

Keywords: symptoms and signs, pulmonary tromboembolism, cardiac failure

\section{Introduction}

Clinical practice is frequently confronted with heart failure of known etiology, complicated with pulmonary thromboembolism (PTE), or with cases in which pulmonary thromboembolism has led to acceleration of heart failure onset. Early diagnosis of pulmonary thromboembolism signs in patients whose prognosis is already aggravated by the condition leading to heart failure onset may make the difference between life and death. This is true under the circumstances in which the percentage of death as a result of untreated pulmonary thromboembolism is high, even in patients with no pre-existent heart condition $[1,2]$. The aim of the study was to assess incidence of pulmonary thromboembolism in patients with cardiac failure and to attempt diagnosis optimization. 


\section{Material and methods}

Study group included 150 patients admitted at Medical Clinic II, Emergency Clinical County Hospital Constanţa with preexistent known or unknown heart disease, which led to cardiac failure. Patients were suspected with incipient pulmonary thromboembolism. The prospective study was conducted in the period 2009-2012.

The study did not include:

- patients with massive thromboembolism followed by heart failure

- patients with right-sided heart failure secondary to thromboembolic pulmonary hypertension

- patients undergoing recent surgery procedure

- prolonged bed-ridden patients

- patients with recent lower-limb fracture

- neoplasm patients

Conditions leading to left-sided heart failure were mitral stenosis, complicated or not with atrial fibrillation, arterial hypertension (HTN), ischemic heart disease with left ventricular dysfunction.

Conditions complicated with right-sided heart failure were chronic pulmonary heart disease and posterior myocardial infarction.

Most patients in the study group suspected with pulmonary thromboembolism presented global heart failure. Conditions leading to cardiac decompensation were: ischemic dilated cardiomyopathy, complicated or not with atrial fibrillation; non-ischemic dilated cardiomyopathy; left-ventricle myocardial infarction with after-effects; and mitral stenosis complicated with atrial fibrillation.

Patients in the study group suspected with pulmonary thromboembolism were examined clinically, biologically, and paraclinically in order to establish certain diagnosis of pulmonary thromboembolism. Biological tests consisted of D-dimer dosage or, in certain instances, fibrin monomer qualitative test.

The following additional tests were administered to the patients: electrocardiography (ECG), cardio-pulmonary x-ray, echocardiography, pulmonary angiography, pulmonary CT-scan with contrast medium, vascular doppler ultrasound of the lower-extremity veins.

Patients undergoing recent surgery (40 days before PTE symptoms onset), bed-ridden patients, patients with recent lower-limb fractures, as well as patients diagnosed with a form of neoplasm were not included in the study group; the reason is that these are high-risk PTE patient groups among the general population, so they could have altered the study results. This study aims to prove the association of cardiac failure and PTE, in the absence of other major PTE risk factors $[3,4]$.

Clinical evaluation: hypothesizing PTE upon patients' clinical examination was most important in study group selection and diagnostic strategy approach; it is well-known that, in $90 \%$ of cases, PTE suspicion is raised by symptoms such as dyspnea, thoracic pain, or syncope. The monitored parameters are indicated in Table I.

Table I. PTE-suggestive symptoms and signs

\begin{tabular}{|c|c|}
\hline Symptoms & $\begin{array}{l}\text { - } \text { Dyspnea } \\
\text { - Thoracic pain } \\
\text { - Hemoptysis } \\
\text { - Syncope } \\
\text { - Cough }\end{array}$ \\
\hline Signs & $\begin{array}{l}\text { - } \text { Tachypnea ( }>20 / \mathrm{min}) \\
\text { - Tachycardia }(>100 / \mathrm{min}) \\
\text { - Signs of deep venous thrombosis } \\
\text { - Fever } \\
\text { - Cyanosis }\end{array}$ \\
\hline Radiological changes & $\begin{array}{l}\text { - } \text { Atelectasis or infiltrations } \\
\text { - Pleural effusion } \\
\text { - } \text { Triangular opacity with thoracic wall basis } \\
\text { (pulmonary infarction) } \\
\text { - Hemidiaphragm elevation } \\
\text { - Hilar artery section }\end{array}$ \\
\hline ECG changes & - Right-ventricle overcharge \\
\hline Blood gas changes & - Hypoxemia \\
\hline $\begin{array}{l}\text { Personal pathological } \\
\text { history }\end{array}$ & $\begin{array}{l}\text { - } \text { Obesity } \\
\text { - Smoking } \\
\text { - History of deep venous thrombosis or PTE } \\
\text { - Diabetes }\end{array}$ \\
\hline
\end{tabular}

Most patients in the study group presenting right-sided and global heart failure events manifested, at a given time, intense dyspnea, with no apparent 
cause and despite being administered appropriate therapy for cardiac failure. Severe dyspnea, with sudden onset of persistent polypnea at rest, was often associated with acute, frequently retrosternal, thoracic pain.

The fact that cardiac therapy itself, respectively diuretic therapy, might have caused PTE onset by increased blood viscosity was not overlooked [5].

Many patients with right-sided or global heart failure presented non-invasive PTE in varying degrees of dramatic symptoms. Although such medium or brief PTE episodes do not affect right-ventricle function in patients with a previously undamaged heart, in patients with pre-existent heart failure they have caused right-ventricle damage [6].

Most patients with left-sided heart failure, either left ventricle dysfunction or mitral valve pathology, presented pulmonary infarction or in situ embolism clinical features.

\section{Results}

Of the 150 patients aged between 30 and 84,96 (64\%) were men and 54 (36\%) were women (Figure $1)$.

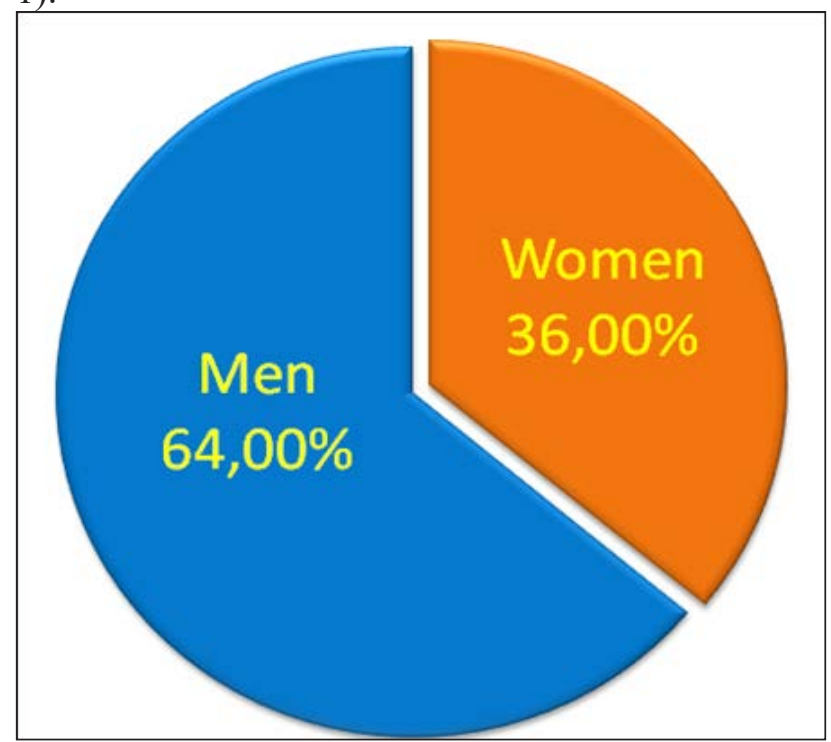

Figure 1. Distribution of cases according to gender
The average age for men was 56.8 years and for women 56.3 years, almost equal values.

Distribution by age and sex is presented in Table II.

Table II. Distribution of the study group by age and sex

\begin{tabular}{|c|c|c|}
\hline $\begin{array}{c}\text { Age group } \\
\text { (years) }\end{array}$ & Males & Females \\
\hline $25-34$ & 1 & 1 \\
\hline $35-44$ & 5 & 3 \\
\hline $45-54$ & 11 & 8 \\
\hline $55-64$ & 19 & 8 \\
\hline $65-74$ & 30 & 14 \\
\hline $75-84$ & 28 & 19 \\
\hline$>85$ & 2 & 1 \\
\hline Total & 94 & 48 \\
\hline
\end{tabular}

The maxium incidence of PTE in heart failure occurs, in men, age range 55-84 years, and women, 65-84 years range, actually following the incidence of heart failure.

We reported the percentage of men and women to all patients and found $\mathrm{p}=0.01$ and $\mathrm{t}=$ 0.23 , meaning that men are statistically significantly correlated with women, in terms of the distribution of the disease (in proportion of $99 \%$ ), so they follow the same distribution.

Patient distribution according to heart failure type is presented in Figure 2.

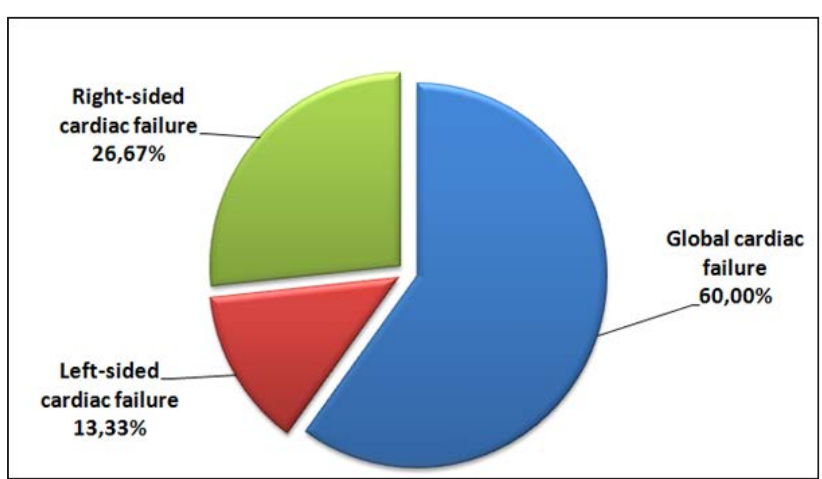

Figure 2. Distribution of cases according to cardiac failure type

Subgroup with left heart failure and PTE 
suspicion, consisted of 20 patients (13.3\%), and they had the following conditions: mitral stenosis complicated with or without atrial fibrillation, ischemic heart disease with left ventricular dysfunction, left ventricular myocardial infarction. There were 12 cases of mitral stenosis, 5 cases of ischemic heart disease and 3 cases of left ventricular myocardial infarction uncomplicated with cardiogenic shock. It is noted that most of this subgoup cases were mitral stenosis $(60 \%)$.

Subgroup of right heart failure and TEP suspicion consisted of 40 patients $(26.7 \%)$ of the initial sample, of which 38 (95\%) were chronic pulmonary heart.

Subgroup of global heart failure and suspected to be with TEP also consisted of 90 patients, meaning $60 \%$ of the initial batch; from these, 62 had ischemic dilated cardiomyopathy (68.8\%), most of them having atrial arrhythmias (atrial fibrillation or atrial flutter).

\section{Discussion}

Suspicion of PTE was initially based on varied symptoms of the patients, sometimes dramatic, marked by syncope and even death, sometimes too little suggestive, consisting of dyspnea and eventualy chest discomfort. Interpretation of the clinical parameters was sometimes difficult because the symptoms and signs given by heart failure itself. All patients had a form of dyspnea (upon exertion and/ or at rest) before the onset of PTE, hemoptysis in a patient with mitral stenosis or with COPD, may be set up only on account of these diseases, and so on.

Dyspnea was present in $99 \%$ cases, i.e. in almost all initial study group patients, except for 3 patients whose symptoms started with syncope. Dyspnea was the most frequent symptom responsible for PTE suspicion. Dyspnea events varied with each case; assessing its etiology was difficult because most heart failure patients presented dyspnea even before PTE onset. PTE-suggestive dyspnea events were the following: suddenly occurring dyspnea at rest, regardless of patient's posture (clinostatic or orthostatic) and increased dyspnea in a patient with previous episodes of dyspnea at rest and/or dyspnea upon exertion.

Isolated dyspnea, sudden apeared, is most often due to a more centrally located PTE, which does not affect the pleura [7]. Often is associated with substernal pain, similar to that of angina pectoris, pain probably due to right ventricular ischemia.

Rarely, dyspnea can install gradually, over several weeks, and the diagnosis of PTE is suggested by the absence of other causes of progressive dyspnea.

Thoracic pain was present in 135 patients in PTE-suspicion study group, i.e. a rate of approximately $90 \%$.

Thoracic pain was manifested in two forms: retrosternal pain, non-aggravated by respiratory movement and acute localized thoracic pain, accompanied by hemoptysis and dyspnea, which suggests pulmonary infarction $[8,9]$.

Chest pain associated with pulmonary embolism is usually sharp and worsens with deep inspiration, cough, and movement, resulting from pleural inflammation in peripheral emboli - pleuritic pain $[10,11]$.

Hemoptysis was present in $72 \%$ of the patients, in most cases associated with dyspnea and thoracic pain. Hemoptysis was low, repetitive, with blood red that after a few days became dark brown. Usually it suggests a small embolism, located distally, near the pleura.

Syncope was present at PTE onset in 3 cases $(2 \%)$; in one case, resuscitation was ineffective and patient exitus occurred as a result of massive PTE; two cases beginning in syncope responded to resuscitation, which was followed by lung CT-scan with contrast medium and catheter thromboembolectomy. Syncope is characteristic for central and massive PTE, with severe hemodynamic repercussions.

Syncope in the onset of pulmonary embolism can be the result of three possible mechanisms. First, greater than $50 \%$ occlusion of the pulmonary vascular tree causes right ventricular failure and impaired left ventricular filling, leading to a reduction in cardiac output, arterial hypotension, reduced cerebral blood flow, and ultimately syncope. The second mechanism of syncope associated with pulmonary embolism is 
the appearance of arrhythmias associated with right ventricular overload. In the third mechanism, the embolism can trigger a vasovagal reflex that leads to neurogenic syncope. However, the contribution of hypoxemia secondary to ventilation or perfusion abnormalities must also be considered and may play an important role in the development of syncope. Moreover, acute pulmonary hypertension may also lead to right-to-left flow across a patent foramen ovale, and thus exacerbate hypoxemia $[12,13]$.

Deep lower-limb venous thrombosis signs were present in 4 patients $(2.6 \%)$. None showed any personal history of deep venous thrombosis (DVT) or PTE.

The association of symptoms and signs is important in clinical practice, assembling the clinical features of frequently encountered PTE syndromes.

Patients showed the following symptom and sign associative types suggestive of massive, submassive, and non-massive PTE:

- Syncope occurred in 3 massive PTE cases $(2 \%)$; in one case, it was followed by patient death and in 2 cases by resuscitation and subsequent hemodynamic stabilization, after pulmonary CT-scan with contrast medium. Death occurred in one male with ischemic dilated cardiomyopathy and atrial fibrillation; the other 2 cases beginning in syncope were 2 males with chronic obstructive pulmonary disease (COPD) and acute myocardial infarction complicated with PTE.

- Intense sudden dyspnea followed by collapse was the onset suggesting massive PTE in 12 cases $(8 \%)$, among which 8 patients with rightsided heart failure (COPD and CPC) and 4 patients with global heart failure (among whom 3 patients with ischemic dilated cardiomyopathy and one patient with mitral stenosis complicated with atrial fibrillation and congestive heart failure).

- Intense sudden dyspnea often associated with tachycardia \pm acute retrosternal pain \pm cyanosis \pm arterial hypertension; these were the clinical features in 22 patients $(15 \%)$. Three of the patients manifesting these clinical features also presented signs of deep venous thrombosis (DVP) in a pelvic limb.
- Sudden dyspnea or aggravation of existing dyspnea associated with acute localized thoracic pain \pm hemoptysis \pm low-grade fever \pm changes in objective lung examination: submatted or matted lung-base, crepitation and/ or sub-crepitation rales, pleural friction rubs at lung base, localized in a certain area; these signs were present in 75 patients $(50 \%)$. Clinical features were consistent with non-massive PTE (pulmonary infarction). Two patients also presented signs of DVP in a pelvic limb.

- Increased cardiac failure events occurred in 38 patients (25\%): dyspnea on more moderate exertion than before, or dyspnea at rest, in a patient who used to present only dyspnea on exertion; increased or persistent hepatic stasis and edema, despite accurate therapy for cardiac failure.

Correlations between significant clinical features and PTE are presented in Table III.

Table III. Correlation between PTE-suggestive clinical features and PTE-grade type

\begin{tabular}{|l|c|c|}
\hline Clinical syndromes & $\begin{array}{c}\text { Symptom } \\
\text { incidence in } \\
\text { study group }\end{array}$ & $\begin{array}{c}\text { Possible PTE } \\
\text { type }\end{array}$ \\
\hline Syncope & $2 \%$ & Massive \\
\hline $\begin{array}{l}\text { Intense sudden } \\
\text { dyspnea, collapse }\end{array}$ & $8 \%$ & Massive \\
\hline $\begin{array}{l}\text { Intense dyspnea, } \\
\text { retrosternal thoracic } \\
\text { pain, tachicardia, } \\
\text { systemic arterial } \\
\text { hypotension, } \\
\text { cyanosis }\end{array}$ & $15 \%$ & Massive \\
\hline $\begin{array}{l}\text { Sudden dyspnea or } \\
\text { aggravated existing } \\
\text { dyspnea, acute } \\
\text { thoracic localized } \\
\text { pain, } \pm \text { hemoptysis } \pm \\
\text { low-grade fever }\end{array}$ & $50 \%$ & $\begin{array}{c}\text { Non-massive } \\
\text { (pulmonary } \\
\text { infarction) }\end{array}$ \\
\hline $\begin{array}{l}\text { Increased cardiac } \\
\text { failure events, } \\
\text { despite appropriate } \\
\text { therapy }\end{array}$ & $25 \%$ & Non-massive \\
\hline
\end{tabular}




\section{Conclusions}

We can conclude that: pulmonary thromboembolism complicates the evolution of clinically manifested cardiac failure or accelerates cardiac failure onset in patients with latent myocardial dysfunction; PTE-suggestive symptoms are dyspnea, with or without tahipnea, thoracic pain, syncope, hemoptysis; these symptoms occur in isolation or associated; dyspnea was the most frequent symptom responsible for PTE suspicion; forms of PTE-suggestive dyspnea were sudden dyspnea and increased dyspnea in a patient with dyspnea at rest; increased cardiac failure events, despite accurate cardiac failure therapy, were correlated at a high rate $(30 \%)$ with non-massive PTE; syncope or shock suggests massive PTE, with severe hemodynamic consequences, such as oliguria, cold extremities and/ or signs of acute right-sided cardiac failure.

\section{References}

1. Stein, P.D., Beemath, A. \& Olson, RE. (2005). Trends in the incidence of pulmonary embolism and deep venous thrombosis in hospitalized patients. Am. J. Cardiol. 95(12), 1525-6. DOI: 10.1016/j.amjcard.2005.02.030

2. Gherasim, L., Iliesiu, A. \& Gherasim, L. (2004). Tromboembolismul pulmonar. In Medicina Interna - Bolile cardiovasculare si metabolice, vol.II, Part II, (pp. 1325-1327). Bucuresti, Romania: Editura Medicala.

3. Kucher, N. \& Golhaber, S.Z. (2005). Management of massive pulmonary embolism. Circulation. 112: e28-e32. DOI: 10.1161/ CIRCULATIONAHA.105.551374

4. Fedullo, P.E., Auger, W.R., Kerr, K.M. \& Rubin, L.J. (2001). Chronic thromboembolic pulmonary hypertension. N. Engl. J. Med. 345(20), 14651472. DOI: 10.1056/NEJMra010902
5. Fedullo, P.F. \& Morris, T.A. (2005). Pulmonary Thromboembolism. In: J.F. Murray \& J.A. Nadel (Eds.), Textbook of respiratory medicine (section L, chapter 48). 4th ed. Elsevier, W.B Saunders Company.

6. Lang, I.M. (2004). Chronic Thromboembolic Pulmonary Hypertension - Not so rare after all. N. Engl. J. Med. 350, 2236-2238. DOI: 10.1056/ NEJMp048088

7. Stein, P. D., Beemath, A., Matta, F., Weg, J.G., Yusen, R.D., Hales, C.A., ... \& Woodard, P. K. (2007). Clinical Characteristics of Patients with Acute Pulmonary Embolism: Data from PIOPED II. The American Journal of Medicine. 120(10): 871-879. DOI: 10.1016/j.amjmed.2007.03.024

8. Ouellette, D. \& Mosenifar, Z. (2015, October). Pulmonary embolism. Retrieved January 29, 2016, from http://emedicine.medscape.com/ article/300901-clinical.

9. Shellhaß, A., Walther, A., Konstantinides, S. \& Bötiger, B.W. (2010). The diagnosis and treatment of acute pulmonary embolism. Dtsch. Arztebl. Int. 107(34-35), 589-95. DOI: 10.3238/ arztebl.2010.0589

10. Agnelli, G. \& Becattini, C. (2010). Acute pulmonary embolism. N. Engl. J. Med. 363, 266274. DOI: 10.1056/NEJMra0907731

11. Riedel, M. (2004). Diagnosing pulmonary embolism. Postgrad. Med. J. 80, 309-319. DOI:10.1136/pgmj.2003.007955

12. Castelli, R., Tarsia, P., Tantardini, C., Pantaleo, G., Guariglia, A., Porro, F. (2003). Syncope in patients with pulmonary embolism: comparison between patients with syncope as the presenting symptom of pulmonary embolism and patients with pulmonary embolism without syncope. Vasc. Med. 8(4), 257-261. DOI: 10.1191/1358863x03vm510oa

13. Dipaola, F., Cucchi, I., Filardo, N., Carnovali, E., Montano, N., Furlan, R. \& Costantino, G. (2006). Syncope as a symptom of non-massive pulmonary embolism: a case report. Intern. Emerg. Med. 1(2), 167-70. DOI: 10.1007/ BF02936550 